\title{
RIESGO DE HELADAS POR INVERSIÓN TÉRMICA EN LA HUERTA DE MURCIA: INCIDENCIA EN LA ACTIVIDAD AGRARIA
}

\author{
David Espín Sánchez \\ Doctorando en el Departamento de Geografía. Universidad de Murcia \\ david.espin1@um.es
}

\section{RESUMEN}

En el Sureste peninsular son frecuentes las situaciones anticiclónicas alimentadas en invierno por advecciones de aire polares/árticas continentales. Bajo estas condiciones, las cuencas y valles fluviales intrabéticos son bastante proclives al desarrollo de inversiones térmicas. Un claro ejemplo lo constituye la Vega Media del Segura en los meses de invierno, con génesis de heladas relativamente frecuentes en sectores localizados. Como complemento al estudio de los factores físicos, se realiza un profundo estudio del parcelario agrícola de la huerta de Murcia y los posibles efectos que puedan generar las bajas temperaturas sobre los principales cultivos del área de estudio, centrados especialmente en los mayoritarios cítricos. Finalmente se proponen una serie medidas anti-heladas activas, pasivas en relación a las características del área de estudio y un sistema empírico de predicción de temperatura mínima, a fin de que los agricultores posean herramientas para mitigar los efectos de las heladas en la Vega Media del Segura.

Palabras clave: Inversión térmica, estrato cálido, Vega Media del Segura, umbral crítico, riesgo, nebulizadores

\section{ABSTRACT}

\section{Frost risk by inversion breakup temperature at the Orchard of Murcia: impact on farming}

In the Southeast peninsular, anticyclonic situations and advections are fed in winter with polar / continental arctic air which is common. Under these conditions, intrabetic basins and river valleys are very prone to the development of inversions breakup temperature. A clear example is the Vega Media of the Segura in winter months, with relatively frequently frosts genesis in localized areas. As a complement to the study of the physical, it is carry out a deep agricultural study of the Huerta de Murcia and the possible effects that can generate low temperatures on the main crops of the study area, with special focus on major citrus. Finally, it is proposed a series anti-frost active and passive in relation to the characteristics of the study area and an empirical system for predicting low temperature, so that farmers possess tools to mitigate the effects of frost in the Vega Media.

Keywords: inversion breakup temperature, wam layer, Vega Media del Segura, critical threshold, risk, nebulizer.

\section{INTRODUCCIÓN}

El presente trabajo tiene la finalidad de abordar uno de los riesgos atmosféricos quizás menos estudiados en el ámbito mediterráneo: las heladas. Por tratarse de un espacio del Sureste Peninsular, y más concretamente de las inmediaciones al área metropolitana de Murcia, el estudio podría carecer de interés debido a la mitificación de bonanza climática que caracteriza climáticamente la zona, pero en detrimento de la lógica, su tipología topográfica unida a determinadas configuraciones sinópticas particulares, propician que las heladas en esta zona -de gran tradición hortofrutícola- sean relevantes, especialmente asociadas a fenómenos de inversiones térmicas. En esta línea, se justifica el interés del estudio, que sin duda es novedoso y pionero en las regiones meridionales de la Península Ibérica. 
Como área de estudio se ha elegido la Vega Media del Segura y sus estribaciones montañosas, ubicada en el sector oriental de la Región de Murcia, y cuya parte de su territorio, se encuentra a lo largo del valle corredor de la Depresión Prelitoral del Guadalentín - Bajo Segura (Sureste peninsular). Ésta es una depresión post-orogénica, rellena de materiales neógenos y cuaternarios (López et al., 1986), con altitudes que varían entre los 30 y $250 \mathrm{~m}$ (Figura 1). El área de estudio limita al norte con una serie de elevaciones modestas, de cotas inferiores a $200 \mathrm{~m}$ (cerros de Espinardo, Cabezo de Torres y Esparragal), y al sur con la parte oriental de la Sierra de Carrascoy, más compacta y entallada (Cabezo del Puerto, 528 m; Relojero, $600 \mathrm{~m}$ ). La base de la depresión es avenada por el Bajo Segura, cuyos continuos aportes sedimentarios en épocas de crecidas ha originado una extensa llanura aluvial (Lillo, 2000).

Figura 1. Localización del área de estudio y de las estaciones meteorológicas utilizadas, con indicación de perfiles topográficos

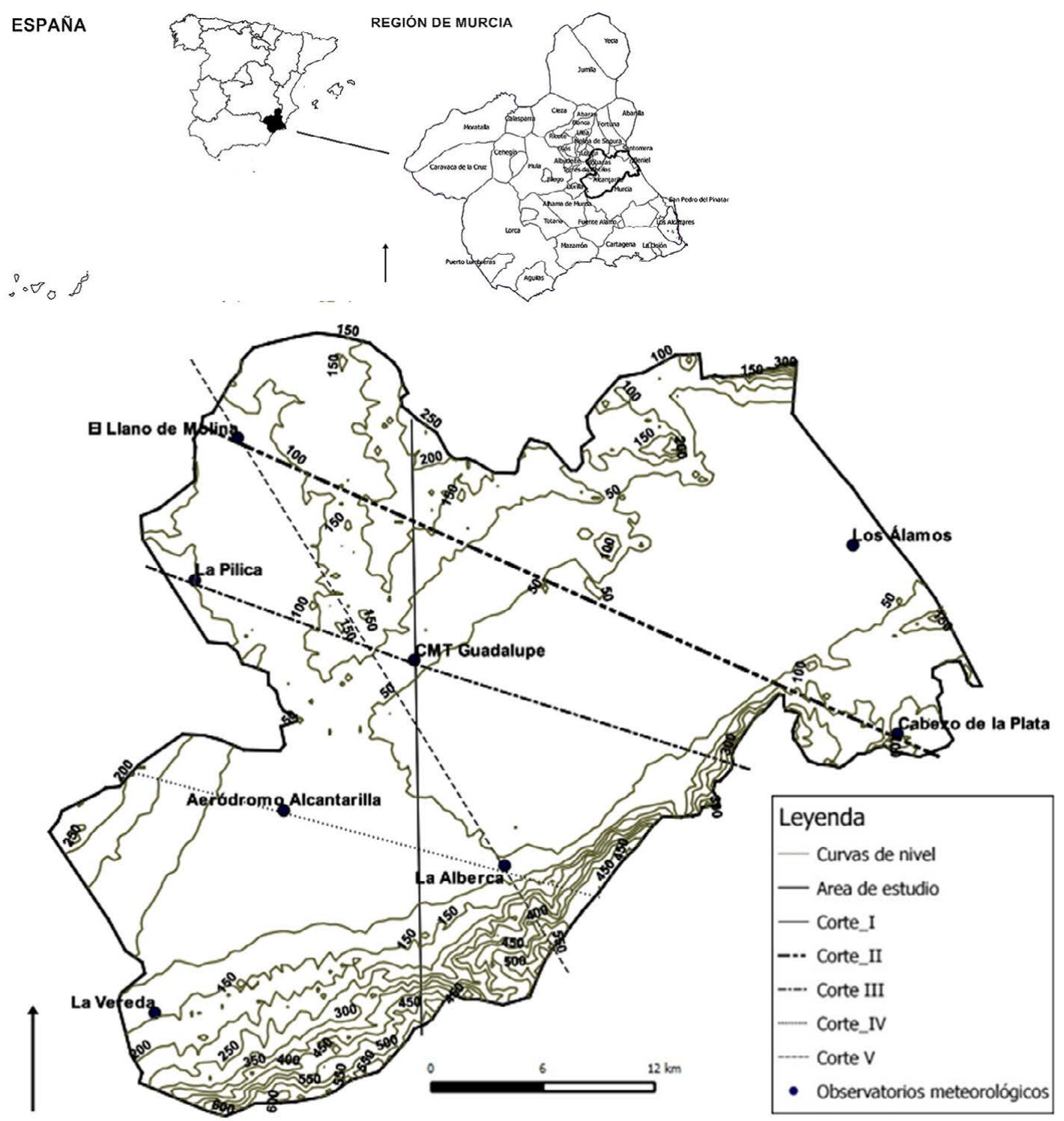

Elaboración propia

La Vega Media del Segura registra un importante número de días despejados y de horas de sol al año, lo que unido al predominio de situaciones anticiclónicas, particularmente en periodo invernal, genera importantes y frecuentes inversiones térmicas por irradiación nocturna (Calvo, 1982; Conesa \& Alonso, 2006). Las heladas, aún sin ostentar una gran importancia como en las comarcas murcianas más occidentales, lo cierto es que la región tampoco está exenta de ellas, con lo que su estudio es trascendental pero a la vez 
complejo. La temperatura media anual se sitúa en torno a $18^{\circ} \mathrm{C}$, las medias de máximas en los meses de verano superan los $32^{\circ} \mathrm{C}$, y las medias de mínimas en los meses de invierno apenas alcanzan los $5^{\circ} \mathrm{C}$. La precipitación anual es escasa (300 a $350 \mathrm{~mm}$ ) y de extrema irregularidad (Geiger, 1973). Con 438.264 habitantes (INE, 2013), la mayoría de los habitantes residen en la ciudad de Murcia, el área metropolitana más poblada de la Región. Las excelentes condiciones agronómicas de sus suelos y la suavidad climática durante gran parte del año han llevado a la población de la Vega a basar su economía tradicional en la actividad agrícola. Las cosechas de plantaciones hortícolas y frutales cítricos han sido exportadas a numerosos países de toda Europa, forjando un sector económico de gran importancia. En los últimos años, dicha actividad agrícola he decrecido de forma significativa (García, 2012), aunque todavía existen muchas pequeñas explotaciones de regadío familiares, que pueden verse afectadas por heladas ocasionales.

\section{METODOLOGÍA}

El estudio de las características térmicas nocturnas en superficie es analizado a través de los registros climáticos de ocho observatorios meteorológicos pertenecientes a la red SIAM (Sistema de Información Agro-meteorológica), del IMIDA, así como a la red de la Agencia Estatal de Meteorología (AEMET). Para el análisis se ha utilizado la serie de datos meteorológicos diarios correspondientes al período de los últimos 15 años (1999-2013), manejando también los datos de los Sondeos Atmosféricos realizados por el Centro Meteorológico Territorial (CMT) de Guadalupe (Murcia) en hora nocturna, concretamente a las 00 horas (00Z).

Para la elaboración de la cartografía de riesgo, se ha trabajado con dos tipos de criterios. En primer lugar, en el relativo a valores físicos, se ha gestionado con Modelo Digital del Terreno (MDT) de la Región de Murcia de 25 píxeles de resolución, que mediante una intersectación con un capa shape lineal del área de estudio, ha generado nuestro soporte físico para el estudio. A continuación se crean varias capas ráster, a las que se añaden atributos de valores medios de días de heladas por meses, $\mathrm{n}^{\circ}$ horas de helada, temperaturas medias de mínimas absolutas...para posteriormente mediante una reclasificación y posterior intersección generar una capa definitiva que atiende a peligrosidad por heladas. Para los criterios de vulnerabilidad y exposición se siguió la misma metodología de reclasificación mediante criterios subjetivos. Las nuevas capas ráster creadas se basaron en criterios de umbrales críticos de temperaturas y valor económico, lo que dio lugar a una capa definitiva de vulnerabilidad por meses (diciembre, enero, febrero y marzo). Finalmente, en una última intersectación se genera la capa final de riesgo por heladas, una vez se superponen los factores que determinan este aspecto.

Como se comentó anteriormente, la generación de un sistema empírico de predicción de temperaturas mínimas, ha centrado buena parte del esfuerzo de este artículo de investigación. Se trata por tanto de un método empírico de regresión múltiple, en los que intervienen factores de temperatura $\left(\mathrm{T}^{\mathrm{a}}\right)$, punto de rocío (Td) y temperatura del bulbo húmedo $(\mathrm{Tw})$. Se establecen por tanto tres zonas diferentes en la aplicación del método, que pueden ser extensibles a zonas homogéneas o principalmente inmediatas (El Llano de Molina, La Alberca y Los Álamos -Beniel-). La ecuación de regresión lineal resultante se obtiene mediante datos de los últimos 15 años, realizándose para cada quincena del periodo correspondiente a Diciembre-Marzo, en los que se obtiene por tanto ocho ecuaciones para cada uno de los sectores.

\section{RESULTADOS Y DISCUSIÓN}

\subsection{Estudio del frío nocturno en base a situaciones de inversión térmica}

El estudio de las situaciones de inversión térmica más importantes producidas en la Vega Media del Segura durante el período 2000-2013 ha permitido extraer interesantes consideraciones, entre ellas las relativas a las condiciones sinópticas que suelen contribuir a su formación y desarrollo. Entre ellas existe un predominio anticiclónico en superficie, alimentado en niveles medios y altos con vaguadas de aire polar/ártica continental, cuya cresta (NE-SO) afecta principalmente a la mitad oriental de la Península Ibérica, con medias muy frías de $-0,6^{\circ} \mathrm{C}$ a $850 \mathrm{hPa}$.

Estos fenómenos de inversión térmica suelen ser más frecuentes durante la estación invernal, coincidiendo con la larga duración de sus noches (14 horas de media sin radiación solar), un débil flujo de viento en superficie (entre 2,2 y $6,5 \mathrm{~km} / \mathrm{h}$ ), y advecciones de componente NO y SO. La temperatura mínima superficial promediada para el conjunto de días de máxima inversión térmica difiere mucho según la posición topográfica de cada observatorio (más de $9^{\circ} \mathrm{C}$ en apenas $116 \mathrm{~m}$ de altitud que separa el fondo del 
valle respecto a las zonas marginales no afectadas). Se trata, por tanto, de inversiones térmicas con estrato cálido muy bajo, con temperatura media de $7,4^{\circ} \mathrm{C}$ a $246,1 \mathrm{~m}$ de altitud y una elevada ratio media de descenso térmico desde el estrato cálido a la superficie $\left(-1,2^{\circ} \mathrm{C} / 100 \mathrm{~m}\right)$. Se comprueba que los valores de temperatura mínima del día de MIT se sitúan por debajo de lo habitual en las zonas de valle, con aparición de heladas frecuentes e intensas (Llano de Molina), y valores muy diferentes en sectores circundantes más altos (Cabezo de la Plata). Las temperaturas máximas absolutas son anormalmente altas para la época del año, generando A.T (amplitudes térmicas) también elevadas en el fondo de valle. Estas características dan lugar a los valores más bajos de temperaturas mínimas en terrenos deprimidos y donde los procesos de inversión térmica (IT) son más importantes. Mientras, las mínimas más elevadas se dan precisamente en enclaves de resalte topográfico, en el eje del piedemonte noroccidental de la Sierra de Carrascoy.

Tabla 1. $\mathrm{N}^{\mathrm{o}}$ medio de días de helada

\begin{tabular}{|c|c|c|c|c|c|c|c|c|c|c|c|c|c|}
\hline & E & $\mathrm{F}$ & $\mathrm{M}$ & $\mathrm{A}$ & $\mathrm{M}$ & $\mathrm{J}$ & $\mathrm{JL}$ & A & $S$ & $\mathrm{O}$ & $\mathrm{N}$ & $\mathrm{D}$ & Total \\
\hline C. de la Plata & 0,2 & 0,1 & * & * & * & * & * & $*$ & * & * & * & 0,1 & 0,4 \\
\hline La Vereda & 0,3 & 0,5 & 0,1 & * & * & * & * & * & * & * & * & 0,2 & 1,1 \\
\hline La Pilica & 0,7 & 0,2 & 0,1 & * & * & * & $*$ & * & * & * & * & 0,3 & 1,3 \\
\hline Murcia CMT & 1,8 & 1,0 & 0,2 & * & * & * & $*$ & * & * & * & 0,1 & 1,3 & 4,4 \\
\hline Alcantarilla & 3,7 & 2,5 & 0,6 & * & * & * & $*$ & $*$ & * & * & 0,4 & 2,5 & 9,7 \\
\hline Los Álamos & 2,2 & 2,6 & 0,6 & * & * & * & * & * & * & * & 0,5 & 1,8 & 7,8 \\
\hline La Alberca & 0,9 & 0,9 & 0,1 & * & * & * & * & $*$ & * & * & 0,1 & 0,6 & 2,7 \\
\hline El Llano & 6,5 & 4,0 & 1,1 & 0,1 & * & * & * & * & * & * & 1,5 & 16,7 & 19,9 \\
\hline
\end{tabular}

Fuente: SIAM-IMIDA y AEMET. Elaboración propia

Por último los vientos de componente $\mathrm{N}$ en el norte de la Vega, explican en parte, que el descenso térmico ejercido en la zona de El Llano de Molina sea más importante que en el resto del área de estudio, y que en otros sectores igualmente deprimidos orográficamente. Los otros observatorios de valle, donde teóricamente las temperaturas deberían ser igual de bajas, se ven influenciados por parámetros ventosos diferentes (Tabla 1).

Tabla 2. Temperaturas mínimas medias diarias $\left({ }^{\circ} \mathrm{C}\right)$ de las SIT analizadas

\begin{tabular}{lccccccccc}
\hline Situaciones SIT & C.Plata & Vereda & Pilica & CMT & Alberca & Alcantarilla & Álamos & El LLano & Dif T \\
\hline 25 ene - 1 feb 2005 & $*$ & 1,4 & 0,3 & $-0,4$ & 0,1 & $-0,2$ & $-0,8$ & $-3,6$ & 5,0 \\
18-25 febrero 2005 & 4,5 & 3,9 & 3,4 & 3,3 & 3,0 & 2,8 & 1,3 & $-0,6$ & 5,1 \\
1-2 febrero 1999 & 0,9 & $-1,1$ & $*$ & $-2,0$ & $-1,9$ & $-4,1$ & $*$ & $-5,6$ & 6,5 \\
24-27 diciembre 2010 & 3,7 & 3,6 & 3,0 & 2,9 & 2,4 & 1,6 & 1,6 & 1,6 & 2,1 \\
22-23 enero 2011 & $-0,7$ & 1,0 & $-1,1$ & $-1,2$ & $-1,7$ & $-3,1$ & $-2,4$ & $-4,2$ & 5,4 \\
15-17 diciembre 2010 & 1,0 & 1,3 & 0,0 & 0,2 & $-0,7$ & $-2,1$ & $-1,7$ & $-3,1$ & 4,4 \\
15-17 febrero 1999 & 3,9 & 2,0 & $*$ & 1,1 & 1,2 & $-0,7$ & $*$ & $-3,1$ & 7,0 \\
2-3 marzo 2004 & 1,6 & 0,6 & 0,6 & $-1,1$ & $-0,6$ & $-1,4$ & $-1,2$ & $-3,8$ & 5,3 \\
8-10 enero 2000 & 4,0 & 2,9 & 2,7 & 1,4 & $*$ & 0,3 & $*$ & $-2,4$ & 6,5 \\
12-16 enero 2003 & 3,1 & 2,0 & 1,3 & 0,4 & 0,3 & $-0,2$ & 0,4 & $-3,4$ & 6,5 \\
15-17 noviembre 2004 & 4,4 & 3,3 & 2,3 & 2,2 & 1,7 & $-0,3$ & $-0,9$ & $-3,1$ & 7,5 \\
16-18 noviembre 2007 & 5,0 & 3,0 & 1,9 & 1,3 & 1,1 & 0,1 & $-0,2$ & $-2,3$ & 7,3 \\
29-31 enero 2006 & 2,9 & 3,7 & 2,8 & 1,8 & 1,8 & 1,9 & 2,6 & 0,8 & 3,0 \\
7-10 enero 2006 & 3,0 & 3,7 & 2,2 & 2,0 & 2,1 & 2,0 & 1,6 & 1,8 & 2,1 \\
29-30 noviembre 2013 & 2,2 & 2,8 & 1,2 & $*$ & $-0,1$ & $*$ & 1,0 & $-3,5$ & 6,3 \\
16-19 diciembre 2001 & 4,6 & 4,2 & 3,8 & 2,7 & 2,9 & 2,3 & 2,3 & 0,7 & 3,9 \\
9-19 febrero 2012 & 2,3 & 2,9 & 1,4 & 0,1 & 0,2 & $-0,8$ & $-1,3$ & $*$ & 4,1 \\
9-10 enero 2009 & 2,4 & 3,3 & 2,3 & 1,2 & 1,9 & 0,3 & 1,0 & $-0,9$ & 4,2 \\
24-26 diciembre 1998 & 5,4 & 2,8 & $*$ & 1,9 & 2,9 & 0,8 & $*$ & $-0,9$ & 6,2 \\
20-28 noviembre 1999 & 5,6 & 4,0 & 4,3 & 2,7 & 3,4 & 2,2 & $*$ & $-0,1$ & 5,6 \\
\hline \multicolumn{1}{c}{ Media } & 3,1 & 2,5 & 1,9 & 1,1 & 1,1 & 0,1 & 0,2 & $-1,9$ & 5,0 \\
\hline
\end{tabular}

Fuente: SIAM-IMIDA y de AEMET. Elaboración propia 


\subsection{Mosaico agrícola de la Huerta de Murcia}

Tras el análisis de los procesos de inversión térmica, junto a los patrones sinópticos que determinan unos bajos valores de temperaturas y génesis de heladas por irradiación, es preciso hacer un estudio y posterior valoración del mosaico agrícola que presenta la Huerta de Murcia. Ésta se va a caracterizar por unas particulares de peligrosidad y vulnerabilidad que darán lugar en última instancia a pérdidas económicas durante las noches de helada relativamente frecuentes. La amalgama de cultivos que presenta la zona de estudio es amplia y compleja, por lo que se hace imprescindible el estudio de umbrales críticos de temperatura, y la ubicación de las diferentes plantaciones en consonancia con los registros de temperatura mínima de cada zona.

Si se analiza el complejo mosaico de variedades de cultivo de la Huerta de Murcia, lo primero que llama la atención en la amplia extensión que posee el tejido agrícola, pues de los $619,1 \mathrm{~km}^{2}$ de superficie total, $277,7 \mathrm{~km}^{2}$ corresponden a tierras de cultivo (44,9\%), que en términos comparativos correspondería al tamaño de términos municipales tales como Águilas, Totana o Fuente Álamo -de los más extensos de España-. Atendiendo a los trabajos de Calvo (1971), hay que considerar que durante los últimos siglos, la Huerta de Murcia ha experimentado un cambio sustancial, evolucionando de una extensión de $82.1 \mathrm{~km}^{2}$ en 1621, 113.4 a finales de siglo XIX, para posteriormente experimentar un importante auge (215,6 en 1970) hasta llegar a $277,7 \mathrm{~km}^{2}$ en el año 2013.

De las actuales 27.774 ha. que componen los cultivos de la zona de estudio, se aprecia un predominio notorio en las tierras de regadío $(86,0 \%)$, aunque es preciso considerar que durante los últimos años -desde 2008- se produce un cambio en el parcelario apostando por cultivos de secano en detrimento del regadío. Así pues, en la actualidad son 23.884 ha de regadío, localizado preferentemente en las depresiones del valle, y 3.890 ha de secano $(14,0 \%)$ que tiene su ubicación en piedemontes y relieves marginales de la zona de estudio (Figura 6).

Figura 2. Superficie regadío/secano de la Huerta de Murcia

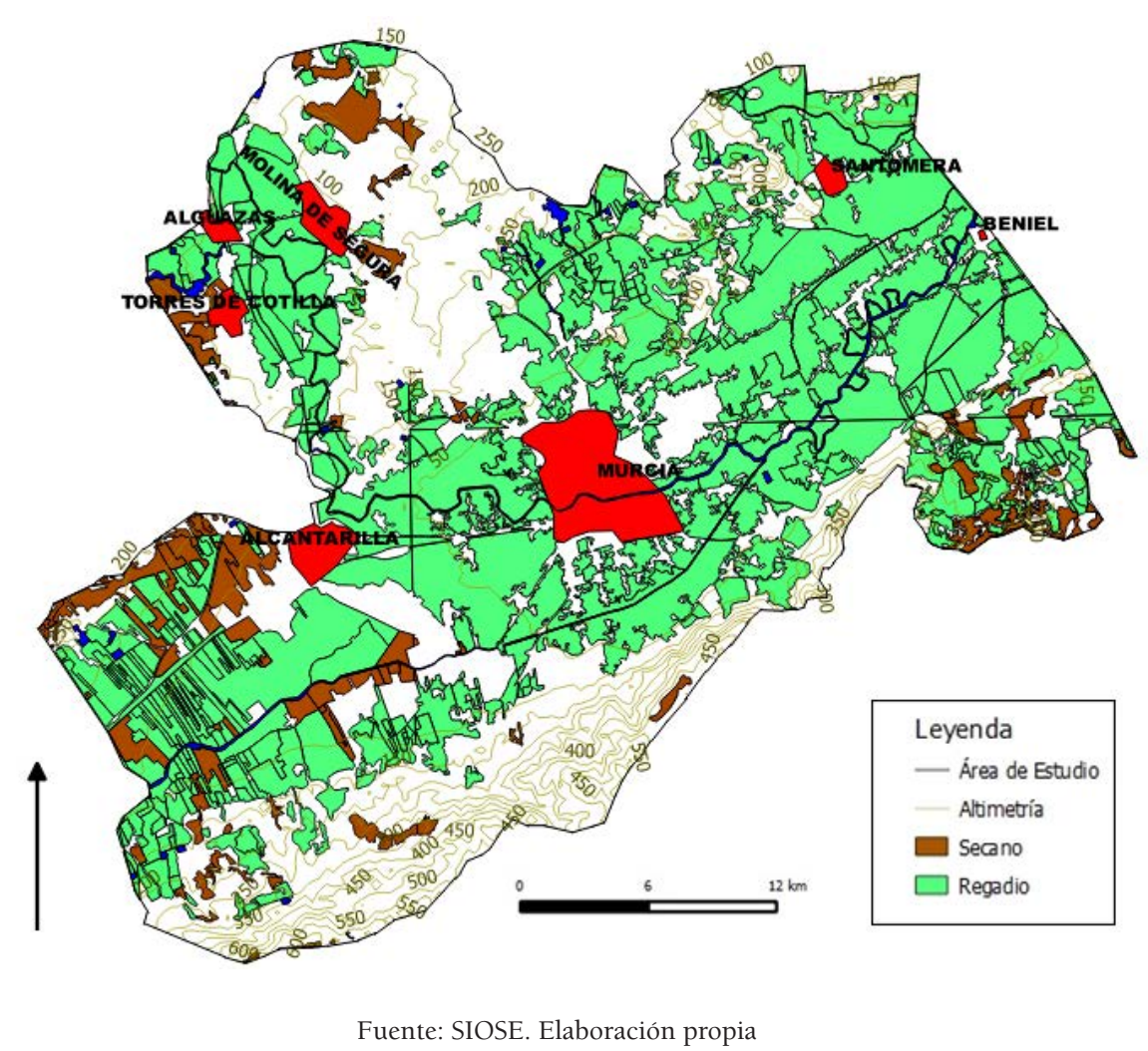

De ellas, un total de 19.550 ha. de regadío del municipio de Murcia distribuyéndose muy desigualmente, cuyas 14.500 ha. se reparten entre seis tipologías aunque con el claro dominio de los cítricos (Andrés Sarasa, 2011). 
Analizando pormenorizadamente las variedades más representativas en la Huerta de Murcia, para posteriormente estudiar su grado de vulnerabilidad frente a las heladas, se advierte que del total cultivado, los que poseen la mayor extensión, son los cultivos leñosos $(77,9 \%)$ frente a los herbáceos $(23,1)$. Analizando en primer lugar al grupo de los herbáceos, el predominio es para las hortalizas con un 48,2\% en su conjunto, con la alcachofa y el melón como especies más cultivadas.

Tras el estudio somero del reparto de los diferentes grupos y especies de cultivo, es preciso valorar en términos porcentuales, que cultivos son los más representados en la Huerta de Murcia. El limonero con un $30,7 \%$ del total de la superficie cultivable, aparece con diferencia, como el cultivo más importante de nuestra zona de estudio, siguiéndole en segunda posición otro cítrico importante, el naranjo con un 22,5\%. Si se le une el mandarino, el grupo de los cítricos representa el 55\% del total, sin duda el grupo más importante en el mosaico de la huerta. Seguidamente aparece el olivo (3,2\%), el melocotonero $(1,9 \%)$. Finalmente y con una extensión bastante más reducida aparecen algunos cultivos hortícolas, como la alcachofa $(1,5 \%)$ y el melón $(1,4 \%)$.

Si se realiza un estudio agrícola pormenorizado del área de estudio se observa una caracterización mucho más compleja, que será vital a la hora de entender la interrelación existente con los rasgos climáticos de la zona. Para el siguiente análisis, se divide la Huerta de Murcia en siete sectores: La Costera sur de Murcia (La Alberca), relieves marginales del Cabezo de la Plata, limonar de Beniel - Santomera, periferia Murcia-Ciudad, corredor de Molina de Segura, Alcantarilla y piedemontes occidentales del Carrascoy (La Vereda).

El ámbito de la Costera sur de Murcia, representado por las pedanías murcianas de Santo Ángel, La Alberca, Patiño...muestra un parcelario de pequeño tamaño, ubicado fundamentalmente por debajo de la isohipsa de los $100 \mathrm{~m}$. de altitud, si bien es cierto que se localizan cultivos en el piedemonte del sector central de la Cresta del Gallo, llegando incluso hasta la cota de $450 \mathrm{~m}$. En este espacio los cultivos herbáceos y los cítricos -representados por el limonero- sobresalen en el parcelario agrícola. Se localizan cultivos herbáceos principalmente en la margen derecha del Canal del Reguerón entre 50 y 100 m. de altitud, ubicándose por tanto en zona de inversión térmica.

En los relieves marginales del Cabezo de la Plata, localizados entre 100 y $200 \mathrm{~m}$. de altitud, aparece representado especialmente los cultivos de secano tales como el almendro, especialmente en el sector más meridional, y el olivo. La localización de este sector evade las negativas consecuencias del fenómeno de inversión térmica, y como se ha tratado en puntos anteriores constituye una de las zonas con las temperaturas mínimas más elevadas de la zona de estudio tal y como muestran los datos meteorológicas de la estación meteorológica homónima, por lo que las heladas son prácticamente inexistentes en este sector. Este hecho ha provocado de manera reciente la proliferación de cultivos que tradicionalmente se han asentado en la vega, como el limón, naranjo y mandarino, que han tenido un auge importante en los últimos años.

Figura3. Extensas plantaciones de limonero en Beniel

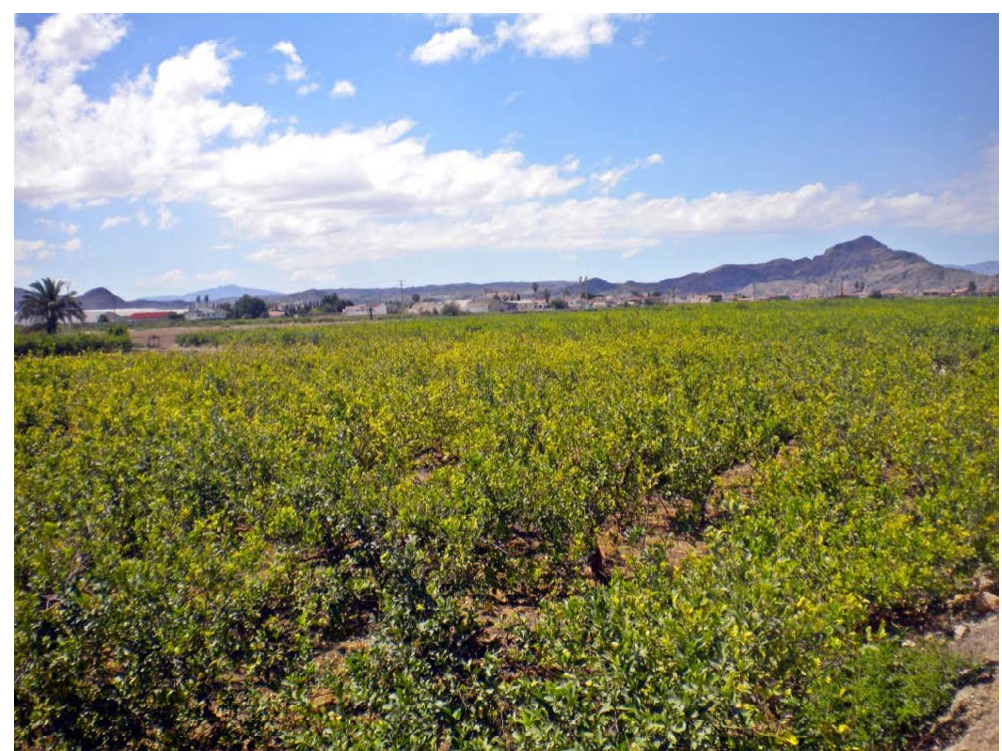


En el sector más oriental de la zona de estudio y limítrofe a la provincia de Alicante y a la Vega Baja del río Segura, aparece el gran limonar del sureste peninsular, que ha sido denominado popularmente como el "limonar de Europa", constituidas en su totalidad por los municipios de Beniel, Santomera y las pedanías más orientales de Murcia, tales como Alquerías o Casillas. Lo primero que destaca en el parcelario agrícola es la supremacía que ejerce el regadío, siendo en parte lógico debido a su ubicación junto al río Segura y en una superficie plana y altitudinalmente inferior a los $50 \mathrm{~m}$. de altitud. El predominio por tanto del limón es incuestionable, abarcando la práctica totalidad de los citados términos municipales, y apareciendo además en los rebordes montañosos del oeste de Santomera, llegando incluso a ascender hasta los $150 \mathrm{~m}$. de altitud, sin duda los más resguardados de las bajas temperaturas.

Los citricultores de Alquerías (Murcia) estiman a pie de campo una recurrencia de daño importante por heladas cada 7-8 años. Tal como ocurre en el presente año (2014) su principal preocupación es la gran sequía que padece la zona, sin lugar a dudas el riesgo climático omnipresente en la actualidad.

En la periferia de Murcia-ciudad, preferentemente en su sector oriental, donde la tradicional huerta en la actualidad se mantiene en un estado aceptable, predominan especialmente los cítricos, con el cultivo del limonero de nuevo sobresaliendo en este sector del área de estudio. A diferencia del área oriental conformada por Beniel-Santomera, en esta zona el parcelario se muestra mucho más fragmentado y de menor extensión, constituyendo en la gran mayoría de las ocasiones plantaciones donde sea alternan los dos cítricos mayoritarios con la tradicional huerta de herbáceos. Además, esta zona asentada ligeramente por debajo del nivel de $50 \mathrm{~m}$. de altitud, padece bajas temperaturas invernales con una media de 10 días de helada al año y temperaturas mínimas absolutas que por término medio suelen descender a $-1,4^{\circ} \mathrm{C}$ en cada invierno.

En la unidad del corredor de Molina de Segura, es preciso comentar que esta zona, es donde se registran los datos de temperatura mínima más bajos de la Huerta de Murcia, con temperaturas medias de las mínimas absolutas de $-3,8^{\circ} \mathrm{C}$. Es decir, los cultivos de la zona están expuestos cada invierno a valores que rondan $-4^{\circ} \mathrm{C}$ con un periodo de retorno bajo, de un año. El microclima generado en este corredor con disposición N-S y encajado entre los relieves periféricos occidentales de Alguazas-Las Torres de Cotillas y los del este de Molina de Segura genera un pasillo por donde un flujo frío y denso del norte con velocidades reducidísimas marca el panorama microclimático de la zona en los procesos de inversión térmica. En una extensión considerable de $50 \mathrm{~km}^{2}$ quedan representados gran multitud de cultivos, sobresaliendo extraordinariamente el cultivo del melocotonero.

Figura 4. Vista panorámica del corredor de Molina de Segura

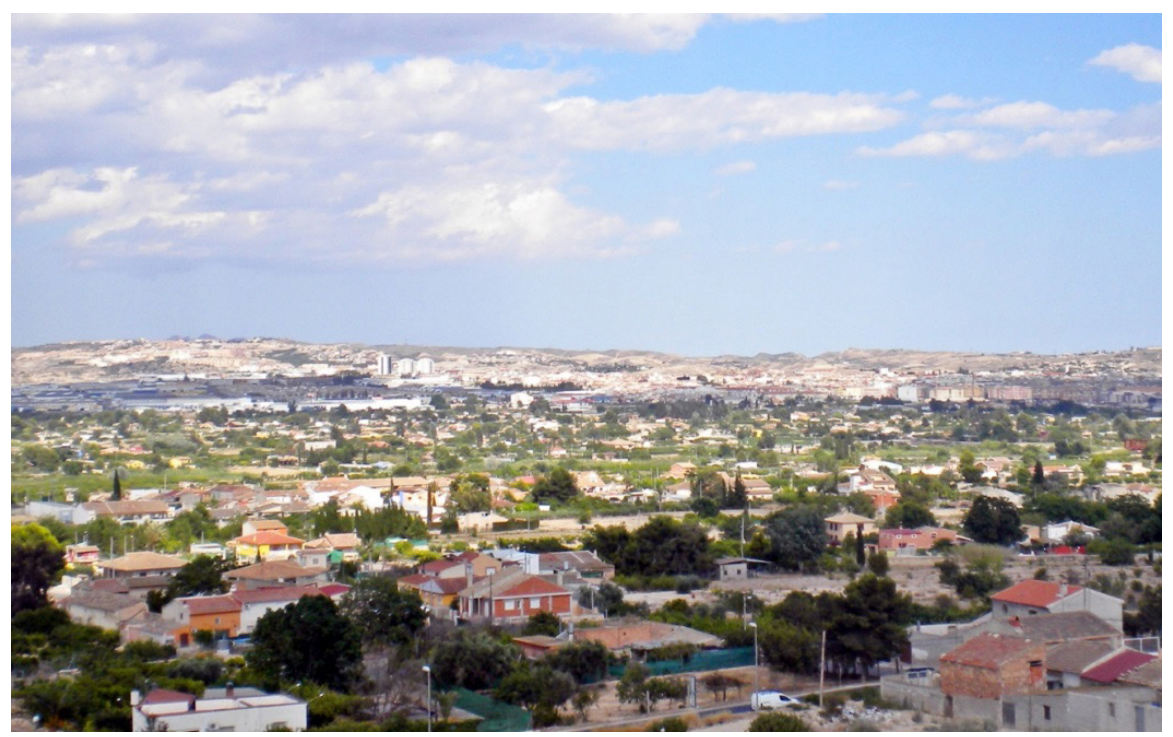

En las inmediaciones de Alcantarilla, y especialmente en el suave piedemonte al oeste del municipio, se asienta una superficie de cultivos caracterizados principalmente por su gran variedad y la particularidad topológica de las parcelas, mayoritariamente rectangulares y muy alargadas en sentido perpendicular 
a las curvas de nivel. Este sector se distribuye en un suave glacis desde 250 a $100 \mathrm{~m}$. de altitud hasta llegar al fondo de valle por donde discurre el Canal del Reguerón. El cultivo predominante de la zona es el almendro, situado especialmente en la zona más distal del glacis y por encima de los $100 \mathrm{~m}$. de altitud. Otro de los cultivos mayoritarios en este sector se corresponde a los herbáceos, ubicados en el inicio de la depresión del Guadalentín, y en la margen izquierda del río.

En los piedemontes más occidentales de Carrascoy, representados por las entidades poblacionales del Molino de la Vereda y las urbanizaciones en "Frondoso Valley" como Torreguil, se desarrolla una amplia variedad de cultivos, todos ellos por encima de $100 \mathrm{~m}$. de altitud y asentados en abanicos, que caracterizan este espacio montañoso.

La puesta en marcha de nuevos cultivos modernizados en uno de los abanicos aluviales del sector oeste de Carrascoy ha reducido drásticamente la vulnerabilidad y exposición de los cultivos de esta zona a las heladas. En efecto, con datos meteorológicos contrastados se determina que en este sector rara vez la temperatura desciende de $0^{\circ} \mathrm{C}$, con lo que el riesgo por helada es de los más bajos de la Huerta de Murcia, junto al Cabezo de la Plata. Se trata por tanto de uno de los métodos pasivos llevados a cabo más relevantes en la zona, en un claro ejemplo de reducción de vulnerabilidad al asentarse en una superficie donde los procesos de inversión térmica no influyen, y por tanto siendo prácticamente nulos los perjuicios por heladas.

\subsection{Umbrales críticos de temperatura según cultivos}

La gran proliferación de los agrios en la zona, bien puede deberse a la gran facilidad de adaptación que poseen los cítricos en climas muy disímiles. A pesar de su gran importancia en la zona de estudio, se encuentran sometidos a temperaturas mínimas invernales, que en ocasiones pueden provocar daños a la planta, con lo cual se hace fundamental determinar cuáles son aquellos umbrales críticos por bajas temperaturas. El limonero, posee un límite térmico no muy amplio, con temperaturas que normalmente por debajo de $-2^{\circ} \mathrm{C}$ empiezan a suponer una restricción en su desarrollo. Es por ello, la especie de cultivo de cítricos más sensible al frío con umbrales críticos que en la Huerta de Murcia se han cifrado entre 0.5 y -1.9 en época de frutación, y por debajo de $-2.8^{\circ} \mathrm{C}$ con riesgo de daño para yemas y flores (Ferreras et al, 2003).

Durante el mes de marzo se han alcanzado valores mínimos absolutos de $-4,9^{\circ} \mathrm{C}$ en El Llano, $-2,9^{\circ} \mathrm{C}$ en Los Álamos (Beniel) o $-2,6^{\circ} \mathrm{C}$ en Alcantarilla. El caso de El Llano de Molina vuelve a destacar, ya que durante el tercer mes del año registra de media 1 día de helada y la media de las mínimas absolutas es negativa $\left(-0,7^{\circ} \mathrm{C}\right)$. Aunque con poca extensión, la superficie de limonero que se encuentra al norte de Molina de Segura y en las inmediaciones de Alguazas presenta una exposición y vulnerabilidad alta. Algo menor es la exposición, aunque contrarrestando con la gran superficie existente, en Beniel-Santomera, con una media de las mínimas absolutas para marzo de $0,4^{\circ} \mathrm{C}$ y con un total de 0,6 días de helada al año.

El cultivo del naranjo, presenta unas condiciones fenológicas muy similares a las del limonero, encontrándose además ubicado en las mismas zonas que el principal cítrico de la Huerta. Durante su época de floración, las temperaturas críticas son algo superiores a las del limonero, con lo que se puede decir que este cultivo presenta más vulnerabilidad debido a que la temperatura a la cual la flor empieza a sufrir daños se sitúa en $-2^{\circ} \mathrm{C}$. Esto daría lugar a que en las zonas anteriormente expuestas como El Llano de Molina, Alcantarilla y Beniel se produzcan más daños al superarse con mayor asiduidad ese valor de temperatura). Se calcula un periodo de retorno para $-2^{\circ} \mathrm{C}$ en el mes de marzo de 10 años en El Llano de Brujas, La Alberca y Alcantarilla. En esta zona no existe prácticamente peligro por las bajas temperaturas durante la frutación (abril), ya que desde que se tienen registros en los últimos 20 años, la temperatura no ha descendido de $4,0^{\circ} \mathrm{C}$. Para concluir con el género de los cítricos, en el caso del mandarino, volvemos a encontrar umbrales críticos para floración y frutación similares a los anteriores cítricos. Así pues, los cultivos más expuestos son los desarrollados en Alguazas, pues soportan temperaturas mínimas equiparables a las registradas por El Llano de Molina (mismo ámbito geográfico).

Abandonando los cultivos cítricos -los mayoritarios en la Huerta de Murcia-, se analiza a continuación la tercera variedad más representada en el área de estudio, el almendro, si obviamos el grupo de los herbáceos (donde están representadas varias especies). Con algo más de 2.300 ha, casi la mayor totalidad de extensión se encuentra ubicada por encima de los $100 \mathrm{~m}$. de altitud, fuera completamente del fondo de valle. Principalmente se ubican en las inmediaciones del Cabezo de la Plata, Alcantarilla y piedemonte noroccidental de Carrascoy. 
El cultivo del olivo, con más de 890 ha. en el área de estudio, supone un 3,2\% de la superficie cultivable, constiuyendo por tanto el $4^{\circ}$ más representado en la Huerta de Murcia. La representación espacial del olivar se desarrolla preferentemente en relieves residuales y periféricos, en sectores entre 100 y $150 \mathrm{~m}$. de altitud, bien representados en el Cabezo de la Plata, noreste de Molina de Segura, oeste de Alcantarilla y piedemonte noroccidental de Carrascoy junto a la Vereda.

El único cultivo arbóreo frutal importante en el área de estudio, el melocotonero, aparece como el $5^{\circ}$ cultivo de mayor extensión en la Huerta de Murcia, ocupando una superficie total de 530,3 ha (1,9\%). Como puede comprobarse en la siguiente figura, su extensión no es significativa, aunque bien es cierto que este cultivo se asienta con importancia en la Vega Alta del Segura, representado especialmente por el municipio de Cieza. Así pues, por proximidad aparecen algunos retazos del melocotonero especialmente en los municipios de Alguazas, Molina de Segura y Torres de Cotillas (Figura 21). Esto implica que en la zona más deprimida, las temperaturas en los meses de enero y febrero puedan alcanzar valores extremos de $-8^{\circ} \mathrm{C}$, con más de 10 días de heladas entre ambos meses, y con medias de las mínimas absolutas de $-3,8^{\circ} \mathrm{C}$ y $-2,1^{\circ} \mathrm{C}$ respectivamente.

Para finalizar con el repaso a los cultivos más extendidos en la Huerta de Murcia, es turno del grupo de los cultivos herbáceos, cuya extensión llega a 6.405 ha, llegando a suponer el 23,1\% del total, si bien es cierto que dentro del grupo se pueden encontrar una gran variedad de cultivos, desde forrajeros, hortalizas o leguminosas. A pesar de no poder aportar datos concretos debido a la inmensa amalgama de cultivos dentro de este grupo, su ubicación total en el fondo de valle determina que los valores de temperatura sean bajas, y se vean expuestos a heladas periódicas.

Figura 5. Mapa de cultivos de la Huerta de Murcia

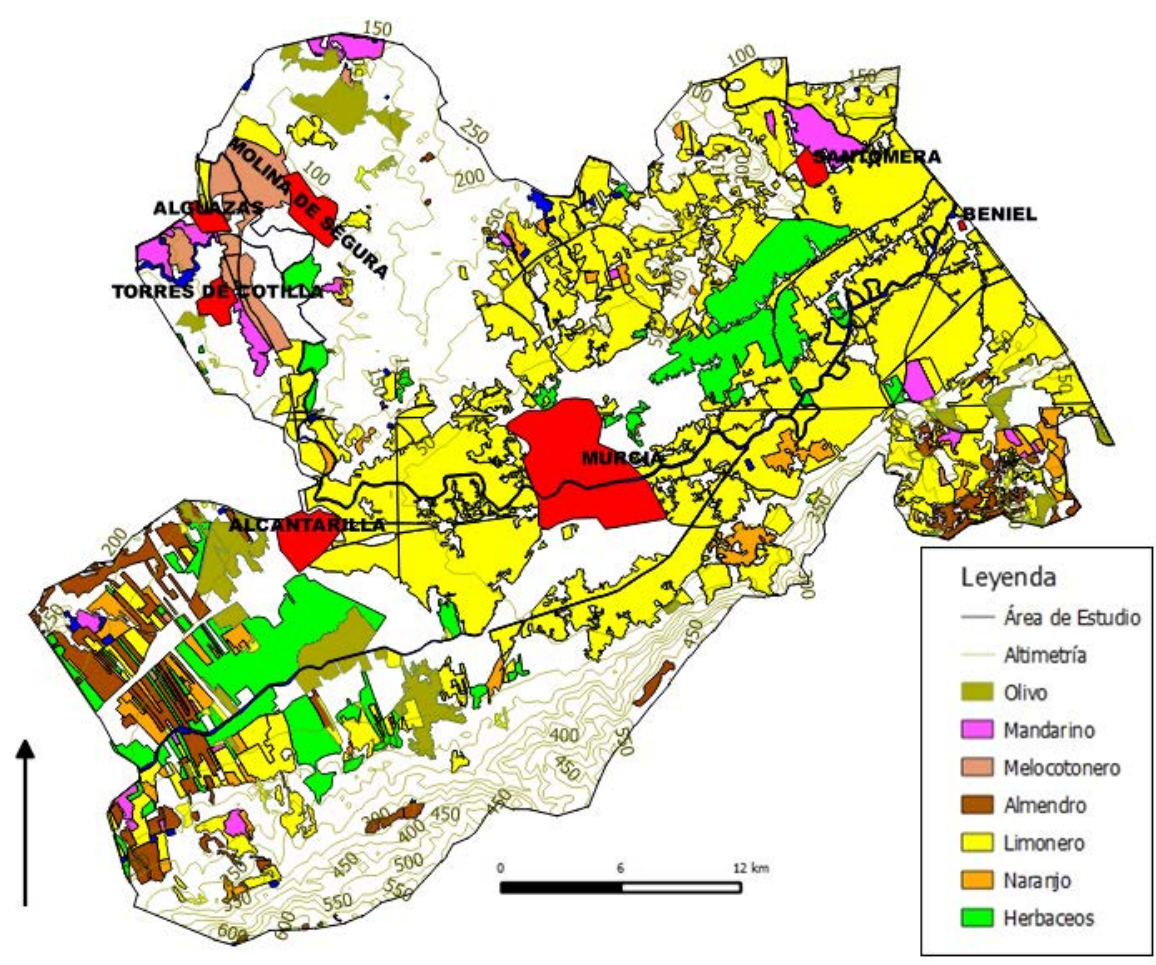

Elaboración propia

\subsection{Propuesta de cartografía de riesgo}

A partir del análisis de la cartografía de riesgo de heladas en la Huerta de Murcia, se pueden obtener datos muy interesantes acerca de los sectores con la mayor probabilidad de pérdidas económicas como consecuencia de la conjunción de una alta vulnerabilidad y peligrosidad por heladas en los meses más fríos del año. 
En el mes de diciembre destaca sobremanera el sector del corredor de Molina de Segura, concretamente al norte de los municipios de Alguazas y Molina de Segura como la zona donde se presenta el mayor riesgo. En general, los mayores índices -riesgo alto y muy alto- se concentran en el sector más septentrional del área de estudio, abarcando también zonas del noreste del casco urbano de Molina. Otros dos sectores aparecen con riesgo medio-alto, situándose al oeste de alcantarilla y en menor medida entre Santomera y Beniel, mientras que por lo general en el resto de la Huerta de Murcia el riesgo es medio-bajo durante el primer mes frío del año en la zona de estudio.

Durante los meses de enero y febrero, con cartografías muy parecidas, vuelve a evidenciarse el mayor protagonismo del corredor del Segura a su paso entre Molina de Segura y Alguazas, aunque con valores muy próximos aparecen zonas del sur de Alcantarilla y Beniel-Santomera con un riesgo alto, explicado en buena parte por una peligrosidad muy alta debido a las bajas temperaturas anteriormente estudiadas. Mientras tanto, se aprecia como durante el mes de febrero se produce un riesgo de helada en la Huerta de Murcia similar al de enero, aunque en esta ocasión más que el factor peligrosidad, el que destaca es el de vulnerabilidad, más elevado en este mes.

Finalmente el mes de marzo aparece como el mes con menor riesgo por heladas en la zona de estudio. A pesar de tratarse con diferencia del mes más vulnerable (debido a los estados fenológicos de floración y primeros frutos), la disminución ostensible de la peligrosidad (donde las heladas empiezan a ser muy esporádicas y débiles) determina unos valores de riesgo bajos. A pesar de ello aparecen zonas muy localizadas en Molina de Segura con riesgo alto, e igualmente al noroeste de Alcantarilla (almendros). Por lo demás, es preciso concluir que el amplio territorio murciano presenta un riesgo medio-bajo (Figura 6).

Figura 6. Mapa de riesgo de helada para diciembre, enero, febrero y marzo

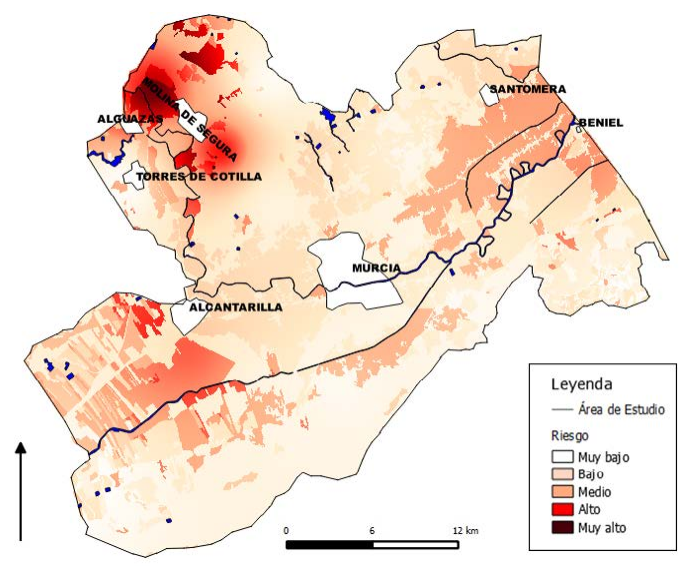

Diciembre

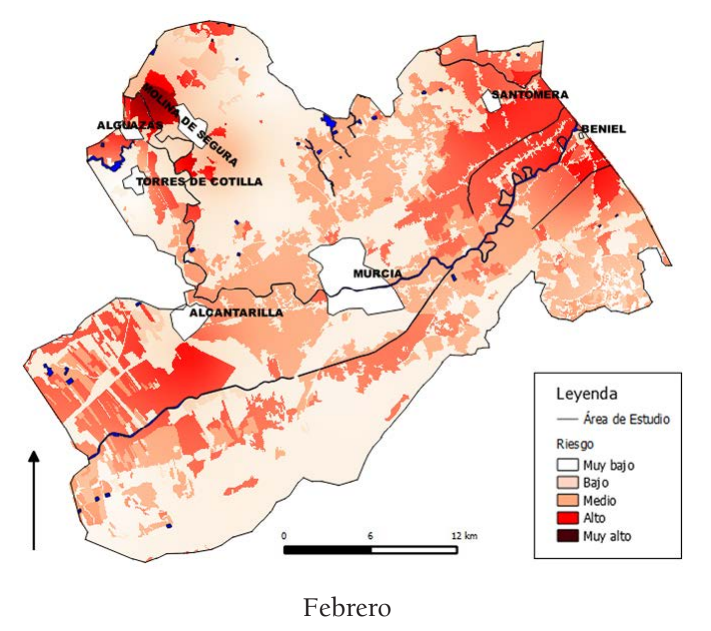

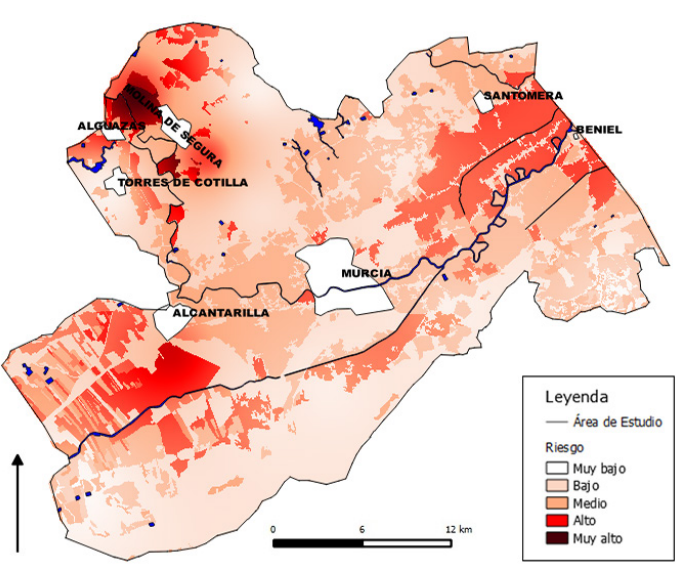

Enero

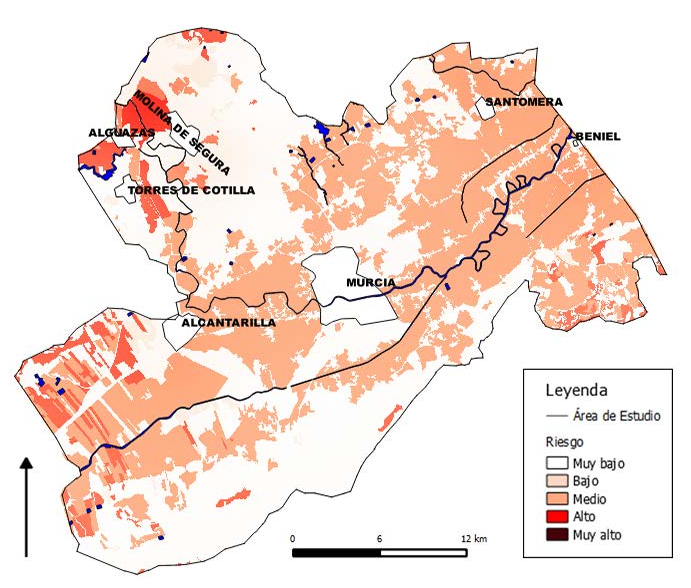

Marzo

Fuente: Elaboración propia 


\subsection{Propuestas de mejoras en la defensa contra heladas}

\subsubsection{Protección anti-heladas adecuadas}

En cualquier zona con tradición agrícola, sus agricultores desde tiempos inmemoriales han tenido que hacer frente a las seculares y siempre presentes heladas, a fin de salvaguardar sus cultivos. En el espacio que nos ocupa, la Huerta de Murcia, debido a la tipología de su parcelario, constituido principalmente de parcelas de pequeñas dimensiones, han sido los métodos pasivos los más utilizados, que además venían a suponer una tecnología más económica que los modernos sistemas anti-heladas de los métodos activos.

En casi todo el área de estudio, exceptuando algunos sectores de Santomera y Beniel, se utilizan procedimientos pasivos para mitigar los efectos de las bajas temperaturas. Uno de los primeros criterios a tener en cuenta es la selección del emplazamiento, ya que en vaguadas o fondo de depresión la acumulación de aire frío será más importante que en los rebordes montañosos. Este factor, presenta una importancia crucial en la zona de estudio, donde se ha demostrado la gran dicotomía existente entre las temperaturas del fondo de valle y los rebordes marginales, cuyas diferencias de temperaturas llegan a suponer hasta $9^{\circ} \mathrm{C}$ en diferencias altitudinales realmente escasas .

Quizás, el aspecto que posea una mayor relevancia en el apartado de medidas pasivas, sea el que tiene en cuenta el drenaje o flujo de aire frío durante la noche, sometido a procesos de inversión térmica. En este sentido, se hace crucial para el agricultor conocer la dirección predominante del flujo a fin de poder controlarlo mediante el uso de vallas, setos o paredes artificiales.... Así pues, en la zona de estudio, se establece un flujo preferente del noroeste tanto en el corredor del Llano de Molina como en las inmediaciones de la ciudad de Murcia. En Alcantarilla lo hace con predominio del oeste, mientras que en las pedanías de la Costera sur (La Alberca), como en Beniel-Santomera se genera un flujo del sur y suroeste respectivamente, al entrar en juego el flujo catabático descendente de la ladera norte de Carrascoy.

Otro aspecto a considerar, es el de la plantación de variedades de cultivos tardíos, cuya floración sea lo más tardía posible, pues en la zona de estudio, a pesar de encontrar una peligrosidad modesta en los meses centrales de invierno (enero, febrero), ésta se reduce enormemente en los meses de marzo y abril, donde ya solo existe cierta peligrosidad en el corredor de Molina de Segura.

En aquellas zonas donde las bajas temperaturas tienen mayor protagonismo y recurrencia, en nuestra caso el corredor del Llano del Molina, y en especial en aquellos sectores donde los agricultores tengan que ocupar el fondo de valle, se recomendaría la puesta en marcha de cultivos intercalados que proporcionarían una radiación de onda larga mayor que desde el cielo. Esta práctica, ha sido ampliamente utilizada en el sur de California con resultados muy aceptables (Snyder, 2010). Entre las variedades más eficientes que podrían tener cabida, se podría considerar a la palmera datilera, que además repercutiría económicamente con la venta de los dátiles, o la aplicada en Alabama (EE.UU) con la implantación de pinos intercalados en este caso entre mandarinos.

Se establece que una buena nutrición y un buen estado sanitario de las plantas favorecen la aclimatación y la resistencia a la congelación (Alden \& Hermann, 1971). Además, una poda favorable también ayuda a atrasar la floración primaveral, por lo que se recomienda en el cultivo de árboles de hoja caduca -por ejemplo en los melocotoneros del corredor del Llano de Molina- retrasar la poda durante los estadios de yema rosa a fin de reducir la muerte invernal de las yemas (Powell \& Himelrick, 2000).

Otra medida económica y de fácil realización por parte del agricultor con el fin de paliar la peligrosidad por helada, sería la de evitar labrar los suelos de los cultivos en invierno y primavera, pues una mayor presencia de porosidad transferirá más el calor debido al bajo calor específico que presentan los suelos. Así pues, Smith (1975) concluyó que era favorable labrar un suelo en otoño para romper los terrones, y posteriormente compactar el suelo, todo ello seguido de un riego.

La eliminación de las cubiertas vegetales en las parcelas cultivadas también supondría una medida pasiva para la lucha contra las heladas. En efecto, la exclusión de las conocidas como "malas hierbas" supondría la disminución de la radiación solar reflejada desde la superficie al exterior. La utilización de un termómetro de infrarrojos, constató que la temperatura superficial de los suelos desnudos estaba entre 1 y $3^{\circ} \mathrm{C}$ más alta que los suelos cubiertos de malas hierbas durante febrero y marzo (Snyder \& Connell, 1993). Esta medida supondría un gran cambio en el parcelario agrícola de la Huerta de Murcia, pues existe la tradición de dejar las malas hierbas en convivencia con los cultivos. 
Si se analiza la utilización de métodos activos, que tienen como base la instalación de tecnología puntera en forma de ventiladores, aspersores, estufas...es preciso comentar que debido al alto costo económico y a la baja rentabilidad de los cultivos parece poco eficiente la instalación de estos sistemas en la Huerta de Murcia. Tan sólo uno de los sectores del área de estudio presenta ciertas garantías a la hora de instalar métodos activos, nos referimos a la extensión de limonar en el sector más oriental, en Beniel y Santomera. Atendiendo a bibliografía internacional y a sistemas ya adoptados y cotejados, quizás el método más eficiente podría recaer en los aspersores. Teniendo en cuenta que nos encontramos en un sector con disponibilidad de agua -río Segura- y que el riego sometido para reducir el daño de helada actuaría para paliar el déficit hídrico de la planta podría ser una buena solución. Quizás para evitar el problema del encharcamiento y consumo de menos agua, cabría la posibilidad de la instalación de nebulizadores. Hay constancia de ser más eficientes en espacios con heladas poco severas y con un flujo ligero de aire frío en noches de inversión.

Con la velocidad de flujo frío medio en este sector, también sería viable la utilización de ventiladores, pues con velocidades inferiores a $2,5 \mathrm{~m} / \mathrm{s}$ obtienen buenos resultados. Además son sistemas que funcionan eficientemente con inversiones térmicas potentes, tal y como se ha podido constatar en el presente trabajo, y en la que el agricultor tendrá que tener en cuenta la dirección del flujo frío predominante, colocando los ventiladores orientados a tal dirección -en este sector del suroeste-.

El último gran sistema de protección activa que tendría cabida analizar en el sector estudiado es el de las estufas de combustible, sin duda el método más eficiente en el control de inversiones térmicas con estrato cálido muy bajo, tal y como es el caso de nuestro estudio (la altura del estrato cálido es de sólo 246,1 m.). A pesar de que este sistema supondría el mayor control de la temperatura mínima en sector más oriental de la Huerta de Murcia, se muestra más eficiente cuanto más frecuentes sean las heladas, y parece además poco viable su aplicación debido al alto costo de la instalación y al requerimiento de una fuente constante de combustible, cuyo precio se antoja complicado para los empresarios y agricultores de la zona.

\subsubsection{Método en el pronóstico de temperaturas mínimas}

Para pronosticar heladas en la Huerta de Murcia, los agricultores deben vivir pendientes de las previsiones meteorológicas que los servicios de meteorología oficiales proporcionan a nivel general. Debido a la baja fiabilidad de éstas, se propone en este apartado, un sistema para la predicción de temperatura mínima en zonas de valle durante madrugadas de total estabilidad (sin viento ni nubosidad) en zonas muy concretas, pues como es sabido, la evolución de la temperatura nocturna difiere extraordinariamente de unos valles a otros. Para ello, se ha utilizado un determinado método empírico a través de ecuaciones de regresión lineal múltiple basada en la ecuación de Allen (1957), utilizando para ello registros de variables meteorológicas (temperatura ambiental y punto de rocío dos horas después de la puesta del sol y finalmente la temperatura mínima observada al final de la madrugada) de los últimos 15 años a fin de obtener las correlaciones más altas.

En el caso del Llano de Molina, se han recopilado un total 259 jornadas con estabilidad atmosférica, obteniendo coeficientes de correlación aceptablemente altos (entre 0,65 y 0,81). Entre ellos destaca la ecuación de la primera quincena de diciembre, que a modo de ejemplo y utilizando para ello 41 jornadas de años anteriores es la siguiente:

A continuación se exponen las ecuaciones resultantes para el resto de las quincenas calculadas, así como el coeficiente de determinación $\mathrm{R}^{2}$ :

$$
\begin{array}{ll}
1^{a} \text { Diciembre: } T_{m}=(0.53 * T)+\left(0.072 * T_{d}\right)+(-4.790) & R^{2}: 0,81 \\
2^{a} \text { Diciembre: } T_{m}=(0.608 * T)+\left(0.043 * T_{d}\right)+(-4.850) & R^{2}: 0,67 \\
1^{a} \text { Enero: } T_{m}=(0.524 * T)+\left(0.066 * T_{d}\right)+(-5.676) & R^{2}: 0,73 \\
2^{a} \text { Enero: } T_{m}=(0.543 * T)+\left(0.046 * T_{d}\right)+(-5.492) & R^{2}: 0,65 \\
1^{a} \text { Febrero: } T_{m}=(0.709 * T)+\left(0.002 * T_{d}\right)+(-6.633) & R^{2}: 0,76 \\
2^{a} \text { Febrero: } T_{m}=(0.594 * T)+\left(0.049 * T_{d}\right)+(-5.338) & R^{2}: 0,75 \\
1^{a} \text { Marzo: } T_{m}=(0.439 * T)+\left(0.182 * T_{d}\right)+(-4.112) & R^{2}: 0,77 \\
2^{a} \text { Marzo: } T_{m}=(0.5 * T)+\left(0.053 * T_{d}\right)+(-3.331) & R^{2}: 0,72
\end{array}
$$

Donde $\mathrm{T}_{\mathrm{m}}=$ Temperatura mínima; $\mathrm{T}=\mathrm{T}^{\mathrm{a}}$ registrada a las dos horas tras la puesta de sol; $\mathrm{T}_{\mathrm{d}}=\mathrm{T}^{\mathrm{a}}$ punto de rocío a las dos horas tras la puesta de sol 


\section{CONCLUSIONES}

A continuación se exponen algunas consideraciones esenciales sobre el contenido del presente artículo científico:

1. En el análisis de la caracterización térmica nocturna del área de estudio, se deduce que las temperaturas mínimas más bajas se registran en aquellos enclaves deprimidos y donde los procesos de inversión térmica (IT) son más notorios. Mientras, las mínimas más elevadas se dan precisamente en enclaves de resalte topográfico, en el eje del piedemonte noroccidental de la Sierra de Carrascoy. Esta distribución espacial de los valores mínimos se explica por frecuentes y potentes procesos de inversión térmica con estrato cálido bajo (a una altitud media de $288,6 \mathrm{~m}$ ) y con gran diferencia térmica con la superficie $\left(2,8^{\circ} \mathrm{C}\right)$, donde la variabilidad del viento debido a la complejidad topográfica de la zona propicia una distribución desigual de los flujos fríos nocturnos

2. En el estudio del parcelario agrícola del área de estudio se concluye que de las actuales 27.774 ha. que compone la extensión de los cultivos de la zona de estudio, se aprecia un claro predominio de las tierras de regadío $(86,0 \%)$, frente a las 3.890 ha. de secano $(14,0 \%)$ que tiene su ubicación en piedemontes y relieves marginales de la zona de estudio importancia en extensión. Los cultivos leñosos $(77,9 \%)$ ejercen una clara supremacía frente a los herbáceos $(23,1)$, éstos últimos representados con la alcachofa y el melón. El limonero con un 30,7\% del total de la superficie cultivable, emerge con diferencia, como el cultivo más importante de nuestra zona de estudio, siguiéndole en segunda posición otro cítrico significativo, el naranjo con un $22,5 \%$. Si se le une el mandarino, el grupo de los cítricos representa el 55\% del total del parcelario agrícola.

3. Se concluye, que la mayoría de los cultivos de regadío -cítricos y herbáceos especialmente- de la zona de estudio se encuentran situados en el fondo de valle, emplazados en zonas de inversión térmica donde las heladas son relativamente asiduas en invierno, mientras que cultivos como el olivo o almendro (secano principalmente) lo hacen en los rebordes montañosos y zonas de piedemonte. En la elaboración de la cartografía de riesgo, donde se han tenido en cuenta criterios de peligrosidad y vulnerabilidad, se pone de manifiesto que los tres sectores con el riesgo más alto en la Vega Media del Segura corresponden especialmente al corredor del río Segura entre Molina de Segura y Alguazas, a los sectores oeste y sur de la cabecera municipal de Alcantarilla y a los cultivos principalmente cítricos ubicados entre los municipios de Santomera y Beniel.

4. Se establecen una serie de propuestas activas y pasivas para la mitigación de las heladas en la zona, que han sido estudiadas teniendo en cuenta las características de la zona de estudio. Entre las medidas pasivas destacan las de la selección del emplazamiento, tener en cuenta el drenaje o flujo de aire frío, la plantación de variedades de cultivos tardíos, la puesta en marcha de cultivos intercalados que proporcionarían una radiación de onda larga, una correcta nutrición y poda del cultivo, evitar labrar los suelos, eliminar las cubiertas vegetales...

En cuanto a métodos activos, debido al alto costo económico y a la baja rentabilidad de los cultivos parece poco eficiente la instalación de estos sistemas en la Huerta de Murcia. Tan sólo uno de los sectores del área de estudio presenta ciertas garantías a la hora de instalar métodos activos (Santomera-Beniel), en este caso el de los aspersores. La utilización más eficiente, dadas sus características, sería la de los nebulizadores. Por último, al contar con una velocidad de flujo frío muy baja en este sector, también sería viable la utilización de ventiladores, que funcionan eficientemente con inversiones térmicas potentes, tal y como se ha podido constatar en el presente artículo. Finalmente, se concluye con la adaptación del método de Allen para la predicción de temperaturas mínimas en sectores localizados, teniendo en cuenta variables ambientales de la zona de estudio.

\section{REFERENCIAS}

ALDEN, J.; HERMANN, R.K. (1971): "Aspects of the cold hardiness mechanisms in plants", en Botanical Review, $\mathrm{n}^{\circ} 37$, pp. 37-142.

ALLEN, C.C. (1957): "A simplified equation for minimum temperature prediction", en Monthly Weather Review, no 85, pp. 119-120.

ANDRÉS SARASA, JOSÉ LUIS (2011): El neopaisaje de la Huerta de Murcia. Junta de Hacendados de la Huerta de Murcia, 196 pp. 
CALVO GARCÍA-TORNEL, F. (1971): "Los cultivos y la estructura agraria en la Huerta de Murcia". Extracto de Tesis Doctoral. Departamento de Geografía. Murcia, 20 pp.

CALVO GARCÍA-TORNEL, F. (1982): Continuidad y cambio en la Huerta de Murcia. Real Academia Alfonso X El Sabio, Murcia.

CONESA GARCÍA, C. y ALONSO SARRIA, F. (2006): "El Clima de la Región de Murcia". En Conesa García, C. (Ed.), El Medio Físico de la Región de Murcia. Cap. 3. Servicio de Publicaciones, Universidad de Murcia. Murcia, pp. 95-127.

FERRERAS FERNÁNDEZ, C.; GARCÍA LIDÓN, A.; PORRAS CASTILLO, I. (2003): "Las heladas en la zona citrícola de la Huerta de Murcia", en Serie técnica y de estudio. Consejería de Agricultura, Agua y Medio Ambiente. Murcia, 96 pp.

GARCÍA MARÍN, FM. (2012): "Dinámicas de crecimiento de la Huerta de Murcia y similitudes con el Véneto italiano". V Jornadas de Introducción a la Investigación de la UPCT, 5; pp. 21-23

GEIGER, F. (1973): "El Sureste español y los problemas de la aridez". Revista de Geografía, vol. 7, n 1-2; pp. 166-209.

I.N.E.: Censo de población y viviendas, 2013. Instituto Nacional de Estadística.

LILLO CARPIO, M. (2000): "La Huerta de Murcia como ejemplo de escorrentía derivada inscrita en la llanura de crecida", enPapeles de Geografía, No 32, pp. 61-75.

LÓPEZ BERMÚDEZ, F.; CALVO GARCÍA TORNEL, F. y MORALES GIL, A. (1986): Geografía de la Región de Murcia. Ketres Editora, Barcelona, 277 pp.

POWELL, A.A.; HIMELRICK, D.G (2000): "Principles of freeze protection for fruit crops", en Alabama Cooperative Extension System, ANR 1057B

SMITH, L.P. (1975): "The modes of agricultural meteorology - Hazards", en Developments in Atmospheric Science, $\mathrm{n}^{\circ}$ 3, pp. 167-171.

SNYDER, R.L.; CONNELL, J.H. (1993): "Ground cover height affects pre-dawn orchard floor temperature", en California Agriculture, n' 47, pp. 9-12.

SNYDER R.L., MELO-ABREU, J.P; VILLAR MIR, J.M. (2010): Protección contra las heladas: fundamentos, prácticas y economía. FAO 2010, 257 pp. 